\title{
GMR
}

\section{Effect of atorvastatin on plasma NT-proBNP and inflammatory cytokine expression in patients with heart failure}

\author{
H.Y. Duan, D.M. Liu, P. Qian, S.L. Wang, L.J. Yan, J.T. Wu, H.T. Yang, X.W. Fan \\ and Y.J. Chu \\ Department of Cardiovascular Medicine, Henan Provincial People's Hospital, \\ Zhengzhou, China \\ Corresponding author: Y.J. Chu \\ E-mail: yingjiechu@yeah.net \\ Genet. Mol. Res. 14 (4): 15739-15748 (2015) \\ Received August 22, 2015 \\ Accepted October 2, 2015 \\ Published December 1, 2015 \\ DOI http://dx.doi.org/10.4238/2015.December.1.25
}

ABSTRACT. The aim of this study was to explore the effect of atorvastatin intervention on plasma N-terminal pro-B-type natriuretic peptide (NTproBNP) and inflammatory cytokine levels in patients with heart failure (HF). One hundred and twenty-three HF patients were selected from our hospital and randomly divided into control $(\mathrm{N}=61)$ and observation $(\mathrm{N}=$ 62) groups; the former received conventional treatment, while the latter were given conventional treatment combined with atorvastatin. Plasma NT-proBNP, inflammatory cytokines [high-sensitive C-reactive protein (hsCRP), interleukin (IL)-6, IL-10] and cardiac function [left ventricular enddiastolic dimension (LVEDD), left ventricular ejection fraction (LVEF), enddiastolic maximum flow rate ratio $(E / A)]$ were compared among groups. The effective rate of treating HF significantly increased after atorvastatin treatment. The plasma NT-proBNP, IL-6, IL-10, hs-CRP, and LVEDD levels significantly decreased $(P<0.05)$, while the LVEF and E/A levels significantly increased $(P<0.05)$ in the observation group compared to the control group and before intervention. The NT-proBNP and cytokine levels significantly differed among patients with different classes of heart function $(P<0.05)$; 
the NT-proBNP and cytokine levels increased with the severity of heart function. Pearson's correlation analysis revealed a negative correlation between the NT-proBNP and inflammatory cytokine levels and LVEF and $E / A$ values, and a positive correlation between these factors and LVEDD $(P<0.05)$. In conclusion, atorvastatin significantly improves cardiac function; the mechanism atorvastatin action was related to the decrease in plasma NT-proBNP and inflammatory cytokine levels.

Key words: Atorvastatin; Heart failure; NT-proBNP; Inflammatory cytokine

\section{INTRODUCTION}

Heart failure (HF) is a cardiovascular disease characterized by abnormalities in ventricular function, specifically left ventricular ejection, caused by injuries in the heart structure. The recent economic development and improvement in the average quality of life has led to a significant increase in the incidence of coronary heart disease and hypertension; this, in turn, has a serious effect on the quality of life, and heavily burdens the social, economic, and medical status of the society (Daida et al., 2012). Recent epidemiological data have shown an HF incidence rate of $2 \%$ in foreign countries, with the incidence rate increasing significantly in elderly patients (up to 10\%). Recent studies conducted in China revealed a 6-fold increase in the mortality rate of patients with HF (Sukpat et al., 2013; Nuzzolo et al., 2014). Therefore, the HF patients must be subjected to early intervention strategies to improve their cardiac function, in order to improve their survival rate and living quality. Statins are lipid-reducing, anti-inflammatory cytokines that exhibit anti-oxidative and immune regulatory properties, and stabilize coronary atherosclerotic plaque, which in turn prevents cardiac remodeling and improves autonomic nerve function (Forteza et al., 2012). Therefore, statin drugs play an important role in curing coronary heart disease and improving heart function. A number of studies have demonstrated that statins help lower the risk of occurrence of clinical adverse cardiovascular events in patients with coronary heart disease, and improve the heart function (Poolsup et al., 2012). Plasma N-terminal pro-B-type natriuretic peptide (NT-proBNP) is known to reflect the degree of myocardial ischemic lesions, and evaluates the functional classification of coronary heart disease. On the other hand, inflammatory cytokines reflect the degree of stability of coronary atherosclerotic plaque (Bracht et al., 2012). Common inflammatory cytokine biomarkers include high-sensitive C-reactive protein (hs-CRP), interleukin-6 (IL-6), and IL-10. So far, very few studies have analyzed (and reported on) the effects of atorvastatin on NT-proBNP and inflammatory cytokines in HF patients. Previous studies have demonstrated the role of statins in reducing the risk of adverse cardiovascular events and improving heart function in patients with coronary heart disease; however, its mechanism of action remains to be clarified. NT-proBNP and inflammatory cytokines play important roles in the pathogenesis of coronary heart disease. Regulating the expression of NT-proBNP and inflammatory cytokines could significantly improve cardiac function. Statins may regulate heart function by influencing the level of expression of NT-proBNP and inflammatory cytokines; however, the exact mechanism remains unclear. This study explored the effect of atorvastatin on plasma NT-proBNP and inflammatory cytokines in HF patients. 


\section{MATERIAL AND METHODS}

\section{Clinical data}

One hundred and twenty-three HF patients admitted to the Cardiology Department of our hospital between January 2012 and June 2014 were selected for this study. The patients were selected based on their conformance with the HF diagnostic criteria detailed in the "Heart Failure Diagnosis and Treatment Guidelines" (Gullestad et al., 2012). The New York Heart Association (NYHA) (Yang et al., 2012) classifies the cardiac function into classes II-IV. The patients who were suffering from an infectious disease or a cerebrovascular disease, those taking immunosuppressive drugs or statins, those with severe liver and kidney failure, chronic lung disease, chronic obstructive pulmonary disease, valvular heart disease, cardiomyopathy, hyperthyroidism, coagulation abnormalities, and mental illness(s) were excluded from this study. The patients were randomly divided into observation and control groups. The observation group comprised 62 cases (38 males and 24 females) aged 35-69 years (mean age: $51.04 \pm 5.21$ years). The underlying disease in these patients ranged from coronary heart disease $(\mathrm{N}=31)$, hypertension $(\mathrm{N}=25)$, and arrhythmia $(N=6)$, while the cardiac function (disease grade) was also distributed among grades II $(N=11)$, III $(\mathrm{N}=36)$, and IV $(\mathrm{N}=15)$. On the other hand, the control group comprised 37 males and 24 females aged $36-65$ years (mean age: $48.22 \pm 5.01$ years). The underlying disease in the members of the control group also ranged from coronary heart disease $(N=28)$, hypertension $(N=27)$, and arrhythmia $(\mathrm{N}=6)$, and the cardiac function was distributed as follows: 11 cases of grade II, 35 cases of grade III, and 15 cases of grade IV. The two groups did not show any statistically significant differences with respect to gender, age, underlying disease, cardiac function, and other general information $(P>0.05)$. This study was conducted in accordance with the declaration of Helsinki, and the methods used in this study were approved by the Ethics Committee of the Henan Provincial People's Hospital. Written informed consent was obtained from all participants.

\section{Treatment methods}

The patients in the control group were not given any statin, while being provided with a specific diet, $\beta$-blockers, nitrates, diuretics, digitalis, angiotensin-converting enzyme inhibitors, and other conventional medical therapy. The observation group was orally administered with $10 \mathrm{mg}$ atorvastatin every night, in addition to being subjected to all therapeutic strategies administered to the control group. The course lasted for 56 nights.

\section{Detection of plasma NT-proBNP and cytokines}

Venous blood samples were collected from the elbows of all patients $(3 \mathrm{~mL})$ before and after treatment, in the morning (fasting conditions). The blood samples were treated with an anticoagulant and subsequently centrifuged at $3000 \mathrm{rpm}$ for $5 \mathrm{~min}$ (within $30 \mathrm{~min}$ ). The separated plasma was analyzed by electrochemiluminescence to detect the plasma NT-proBNP content in an E170 immunoassay analyzer (Hoffmann-LaRoche, Basel, Switzerland). The analysis was completed within 2 days. The plasma hs-CRP was analyzed using a particle-enhanced immunoturbidimetric method, using the 7060 Automatic Biochemical Analyzer (Hitachi Ltd., Tokyo, Japan). Serum samples were obtained by centrifugation at $3000 \mathrm{rpm}$ for $15 \mathrm{~min}$. The IL-6 and IL-10 levels were detected by enzyme-linked immunosorbent assay (ELISA). The ELISA kit was purchased from 
Xinran Biotechnology Company (Shanghai, China). All experiments were performed according to the protocols provided by the manufacturer(s).

\section{Echocardiography detection}

All patients were administered with bedside echocardiography, using the SC-2000 Color Doppler ultrasound diagnostic apparatus (Siemens, Munich, Germany) before and after statin treatment at the left lateral position. The ultrasonic probe was adjusted to a frequency of 2.0-2.5 $\mathrm{MHz}$, and a scanning speed of $50 \mathrm{~mm} / \mathrm{s}$. The apical four-chamber view, parasternal long axis view, and left ventricular long axis view were measured in order to know the left ventricular internal dimension, left ventricular end-diastolic dimension (LVEDD), left ventricular end-diastolic volume index (LVEDVI), left ventricular end-systolic volume index (LVESVI), the left ventricular ejection fraction [LVEF = $($ LVEDVI - LVESVI) $/$ LVEDVI $\times 100 \%$ ], and the maximum flow rate of early diastolic and end-diastolic maximum flow rate ratio $(E / A)$, with reference to the recommended checking points on echocardiography provided by the American Society of Echocardiography.

\section{Observation indexes}

The NT-proBNP and cytokine (hs-CRP, IL-6, IL-10) content and the cardiac function (LVEDD, LVEF, E/A) observed in the plasma of patients between the two groups were compared.

\section{Clinical efficacy}

According to the degree of improvement in cardiac function and clinical research criteria for the assessment of drug guidelines, the treatment was classified into greatly effective (reduction in NYHA cardiac class $\geq 2$ ), effective (reduction in NYHA cardiac class by 1 ), and non-effective (NYHA cardiac class remains unchanged or increases). The patients were also classified according to the NYHA cardiac function class, into class I (presence of heart disease, but an almost unaffected daily life; physical activities do not cause heart palpitations, shortness of breath, and angina attack), class II (presence of heart disease, mildly affected by physical activities, no obvious symptoms at rest, normal physical activities will cause palpitations, shortness of breath, and angina attack), class III (presence of heart disease, physical activities are severely affected; no obvious symptoms at rest, but light physical activities cause palpitations, shortness of breath, and angina attack), and class IV (presence of heart disease; cannot bear physical activity; palpitations, shortness of breath, and angina onset at rest, increasing with physical activities).

\section{Statistical analysis}

All data was analyzed using the SPSS (v.18.0) statistical software (SPSS Inc, Chicago, IL, USA). Statistical data between two groups were compared using the $t$-test. Statistical data were compared among multiple groups by analysis of variance (ANOVA), and reported as means \pm SD. The categorical data between two groups were analyzed using the $\chi^{2}$ test. Pearson's correlation analysis was used to analyze the relationship between plasma NTproBNP and serum cytokine levels and cardiac function indicators. $P$ values $<0.05$ indicated a statistically significant difference. 


\section{RESULTS}

\section{Comparison of the clinical efficacy of the two groups}

The efficacy in the observation group was significantly higher than that in the control group. The difference was found to be statistically significant $(P<0.05$; Table 1$)$.

Table 1. Comparison of clinical efficacy among the two groups.
\begin{tabular}{lccccr}
\hline Groups & Cases & Great effective & Effective & Non-effective & Effectiveness (\%) \\
\hline Observation group & 62 & 26 & 30 & 6 & 90.32 \\
Control group & 61 & 23 & 25 & 13 & 78.69 \\
$\chi^{2}$ & & & & 3.96 \\
$\mathrm{P}$ & & & $<0.05$ \\
\hline
\end{tabular}

\section{Comparison of plasma NT-proBNP and inflammatory cytokine levels between the two groups}

We observed no statistical differences $(P>0.05)$ in the plasma NT-proBNP and inflammatory cytokine (IL-6, IL-10, and hs-CRP) levels among the observation and control groups before treatment. The levels of both indicators were significantly lower in all patients after the treatment, which indicated a significant statistical difference $(P<0.05)$. Furthermore, the indicators were significantly lower in the observation group than those in the control group $(P<0.05$; Table 2).

\section{Comparison of cardiac function in the two groups}

The cardiac function (LVEDD, LVEF, and E/A) of the patients in the two groups did not differ significantly $(P>0.05)$ before treatment. The LVEDD of all patients was significantly lower, while their LVEF and E/A values were significantly higher after treatment (than those before treatment) $(P<0.05)$. The LVEDD values in the patients of the observation group was significantly lower than that in the control group, while the LVEF and E/A values were significantly higher, which also indicated a significant statistical difference $(P<0.05$; Table 3$)$.

\section{Relationship between plasma NT-proBNP, serum hs-CRP, IL-6, and IL-10, and cardiac function}

We observed that the plasma NT-proBNP, serum hs-CRP, IL-6, and IL-10 levels were higher when the cardiac function class was higher prior to the treatment. We observed significant statistical differences between patients showing different cardiac function classes $(P<0.05$; Table 4). The Pearson correlation analysis revealed a positive correlation between the plasma NTproBNP, serum hs-CRP, IL-6, and IL-10 levels and LVEDD ( $r=0.512, P=0.004)$, and a negative correlation between these factors and LVEF and E/A ( $r=-0.602$ and $-0.541, P=0.003$ and 0.005, respectively). 


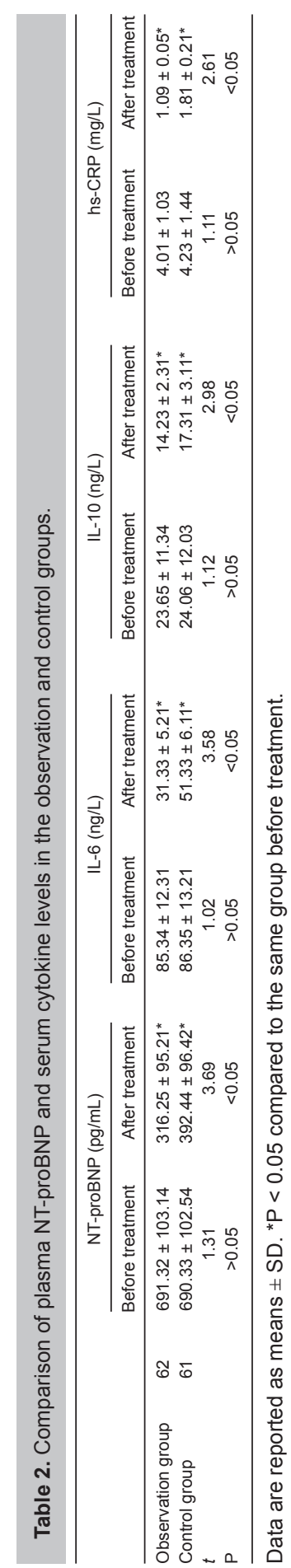


Table 3. Comparison of cardiac function among the observation and control groups.

\begin{tabular}{|c|c|c|c|c|c|c|c|}
\hline & & \multicolumn{2}{|c|}{ LVEDD (mm) } & \multicolumn{2}{|c|}{$\operatorname{LVEF}(\%)$} & \multicolumn{2}{|c|}{$E / A$} \\
\hline & & Before treatment & After treatment & Before treatment & After treatment & Before treatment & After treatment \\
\hline Observation group & 62 & $49.32 \pm 11.30$ & $38.02 \pm 8.31$ & $38.21 \pm 4.31$ & $45.62 \pm 2.01$ & $1.02 \pm 0.08$ & $1.41 \pm 0.04$ \\
\hline Control group & 61 & $48.67 \pm 10.36$ & $40.33 \pm 9.12$ & $39.02 \pm 5.11$ & $41.01 \pm .03$ & $1.01 \pm 0.07$ & $1.09 \pm 1.05$ \\
\hline$t$ & & 1.21 & 3.21 & 1.31 & 2.61 & 0.98 & 2.53 \\
\hline$P$ & & $>0.05$ & $<0.05$ & $>0.05$ & $<0.05$ & $>0.05$ & $<0.05$ \\
\hline
\end{tabular}

Data are reported as means \pm SD.

Table 4. Comparison of plasma NT-proBNP and cytokine levels in different cardiac function classes.

\begin{tabular}{lccccc}
\hline Cardiac function & Cases & NT-pro BNP $(\mathrm{pg} / \mathrm{mL})$ & $\mathrm{IL}-6(\mathrm{ng} / \mathrm{L})$ & IL-10 $(\mathrm{ng} / \mathrm{L})$ & $\mathrm{hs}-\mathrm{CRP}(\mathrm{mg} / \mathrm{L})$ \\
\hline Class II & 22 & $382.11 \pm 65.21$ & $41.36 \pm 10.31$ & $12.08 \pm 5.12$ & $2.31 \pm 0.14$ \\
Class III & 71 & $684.25 \pm 81.31^{\#}$ & $86.24 \pm 14.31^{\#}$ & $24.06 \pm 5.41^{\#}$ & $4.13 \pm 0.21^{\#}$ \\
Class IV & 30 & $901.33 \pm 103.65^{*}$ & $119.21 \pm 15.21^{*}$ & $34.21 \pm 6.01^{*}$ & $6.24 \pm 1.51^{*}$ \\
F & & 5.31 & 4.98 & 5.01 & 4.02 \\
P & & $<0.05$ & $<0.05$ & $<0.05$ & $<0.05$ \\
\hline
\end{tabular}

Data are reported as means $\pm \mathrm{SD} .{ }^{*} \mathrm{P}<0.05$ compared to the cardiac function classes II and III; ${ }^{\mathrm{P}}<0.05$ compared to the cardiac function class II.

\section{DISCUSSION}

Atorvastatin regulates the inflammatory reaction by suppressing the expression of inflammatory cytokines in the immune cells. Previous studies have shown that atorvastatin reverses atherosclerosis plaque. This effect was also found to be stronger than that of pravastatin, simvastatin, and other lipid-lowering statins; this could be because atorvastatin specifically reduces the degree of expression of inflammatory cytokines. In addition, atorvastatin effectively reduces the levels of low-density lipoprotein, total cholesterol, and triglycerides in patients with acute coronary syndrome, by selectively inhibiting the HMG-CoA reductase, reducing the cholesterol synthesis of the liver, and reversing dyslipidemia. Dyslipidemia is the major pathogenic basis of coronary atherosclerotic heart disease (CAHD). Improvements in the blood lipid level of patients with CAHD could stabilize the coronary atherosclerotic hardening plate, recover coronary vascular elasticity, enhance the left ventricular systolic function, improve the heart function, and reduce the risk of occurrence of clinical cardiovascular adverse events. Therefore, atorvastatin has important therapeutic value against coronary heart disease and heart failure. In addition, the degree of atherosclerosis is correlated with the cardiac function and the inflammatory cytokine content in the body (Van Tassell et al., 2012). Severe coronary atherosclerosis is an important cause inducing heart failure. Many clinical studies have demonstrated an increase in the levels of inflammatory cytokines in HF patients. Inflammatory reaction is a major pathogenesis of HF, which is known to change dynamically with the occurrence and development of HF (Gupta et al., 2012). Inflammatory cytokines also prominently participate in myocardial fibrosis and cardiac remodeling, by inducing apoptosis in the myocardial cells, decreasing myocardial systolic function, and decreasing myocardial compliance. Therefore, the severity of HF is obviously correlated with the inflammatory cytokine content. hsCRP, IL-6, and IL-10 are common inflammatory cytokines. Very few intervention studies have analyzed the effect of atorvastatin on inflammatory cytokines in HF patients. A number of studies have demonstrated the major role played by NT-ProBNP in evaluating the change in the degree of heart function in patients with heart failure. NT-ProBNP is a one-peptide hormone biomarker, which plays 
an important role in the diagnosis and severity assessment of cardiovascular disease (Falkensammer et al., 2014). NT-ProBNP is mainly affected by the body blood volume. Lowering of the blood volume or occurrence of myocardial ischemia leads to stimulation of the cardiac muscle cells to synthesize pre-pro-B type natriuretic peptide (pre-ProBNP). The pre-ProBNP is then decomposed into NT-ProBNP and BNP. The half-life of NT-ProBNP is approximately $1.5 \mathrm{~h}$, while that of BNP is about $20 \mathrm{~min}$. Therefore, we observe higher concentrations of plasma NT-ProBNP. In vitro studies revealed that the stability of NT-ProBNP was significantly better than that of BNP, which could also be maintained for 3 days at room temperature (Shafi et al., 2014). Therefore, NT-ProBNP has shown potential applicability in in vitro studies and clinical trials. NT-ProBNP is used to evaluate HF. NT-proBNP levels lower than $100 \mathrm{pg} / \mathrm{mL}$ suggest a low probability of occurrence of HF, while levels higher than $500 \mathrm{pg} / \mathrm{mL}$ suggest a high possibility of occurrence of HF (Eurlings et al., 2014). The results of some previous studies have shown that atorvastatin may inhibit the levels of NT-proBNP and inflammatory cytokines (IL-6, IL-10, and hs-CRP) by stabilizing the coronary atherosclerotic plaque. However, studies evaluating the influence of atorvastatin on NT-proBNP and inflammatory cytokines in HF patients (and its mechanism) are rare. Therefore, atorvastatin may have a significant effect on plasma NT-proBNP and inflammatory cytokine levels.

Our results revealed a significant increase in the effective rate, LVEF, and E/A of HF patients, and a significant decrease in the plasma NT-proBNP, IL-6, IL-10, and hs-CRP levels, and LVEDD after atorvastatin-combined treatment. The level of NT-proBNP and inflammatory cytokines increased with the severity of the heart function. The NT-proBNP and inflammatory cytokine levels were negatively correlated with LVEF and E/A values, and positively correlated with LVEDD. Our results suggested that the atorvastatin intervention had a significant effect on the plasma NT-proBNP and inflammatory cytokine levels. Myocardial remodeling is a major cause of heart failure, with the decrease in myocardial cells leading to the changes in the ventricular function or structure. Inflammatory cytokines promote apoptosis in myocardial cells (thereby decreasing the myocardial cell number) and reduce the myocardial systolic function, which leads to hemodynamic instability, and promote the synthesis of NT-ProBNP in myocardial cells (Mordi et al., 2014). Therefore, one can observe a significant increase in the levels of NT-proBNP, IL-6, IL-10, and hs-CRP in HF patients. We observed a significant reduction in the left ventricular systolic function in HF patients, which in turn significantly decreases the LVEF. Atorvastatin is known to effectively stabilize atherosclerotic plaque, and has strong anti-oxidant and anti-inflammatory functions, which improves the ventricular remodeling and the hemodynamic stability (Kesavan et al., 2014). Therefore, atorvastatin can reverse the abnormal changes in NT-proBNP, IL-6, and IL-10 to some extent. In addition, atorvastatin significantly improves the degree of ventricular remodeling and heart function. Our results suggested that atorvastatin may improve HF by decreasing the plasma NT-proBNP, IL-6, IL-10, and hs-CRP levels; this observation was consistent with those of previous studies, which revealed a significant correlation between atorvastatin and NT-proBNP, IL-6, IL-10, and hs-CRP (Bennaceur et al., 2014; Zhang et al., 2014). In addition, a previous study has shown that the atorvastatin-induced improvement in heart function (in HF patients) is unaffected by the blood lipid level and improvements in the degree of coronary lesion degree (Sarath et al., 2014), which analyzed the role of atorvastatin in reducing low-density lipoprotein cholesterol levels and increasing high-density lipoprotein (HDL) levels (Xu et al., 2014). However, the mechanism with which atorvastatin improves the degree of coronary stenosis must be studied further. Our research demonstrated that the levels of NT-proBNP, IL-6, IL-10, and hs-CRP played an important role in evaluating the cardiac function. Atorvastatin intervention may decrease the levels of NT-proBNP and inflammatory cytokines to improve the heart function and the treatment effect in patients with 
HF. Left ventricular remodeling is a major pathogenetic basis of HF (Klop et al., 2014); however, the role of NT-proBNP, IL-6, IL-10, and hs-CRP in left ventricular remodeling has not been explored here, and requires further study.

In summary, atorvastatin significantly improves the cardiac function, the mechanism of which may be related to the decrease in plasma NT-proBNP and inflammatory cytokine levels in the patient body.

\section{Conflicts of interest}

The authors declare no conflict of interest.

\section{REFERENCES}

Bennaceur K, Atwill M, Al Zhrany N, Hoffmann J, et al. (2014). Atorvastatin induces T cell proliferation by a telomerase reverse transcriptase (TERT) mediated mechanism. Atherosclerosis 236: 312-320.

Bracht L, Barbosa CP, Caparroz-Assef SM, Cuman RK, et al. (2012). Effects of simvastatin, atorvastatin, ezetimibe, and ezetimibe + simvastatin combination on the inflammatory process and on the liver metabolic changes of arthritic rats. Fundam. Clin. Pharmacol. 26: 722-734.

Daida H, Takayama T, Hiro T, Yamagishi M, et al. (2012). High HbA1C levels correlate with reduced plaque regression during statin treatment in patients with stable coronary artery disease: results of the coronary atherosclerosis study measuring effects of rosuvastatin using intravascular ultrasound in Japanese subjects (COSMOS). Cardiovasc. Diabetol. 11: 87.

Eurlings LW, Sanders-van Wijk S, van Kraaij DJ, van Kimmenade R, et al. (2014). Risk-stratification by serial NT-proBNP measurements during admission and early after discharge in heart failure patients. J. Card. Fail. 215: 1031-1042.

Falkensammer J, Frech A, Duschek N, Gasteiger S, et al. (2014). Prognostic relevance of ischemia-modified albumin and NTproBNP in patients with peripheral arterial occlusive disease. Clin. Chim. Acta 438C: 255-260.

Forteza A, Romano JG, Campo-Bustillo I, Campo N, et al. (2012). High-dose atorvastatin enhances impaired cerebral vasomotor reactivity. J. Stroke Cerebrovasc. Dis. 21: 487-492.

Gullestad L, Ueland T, Vinge LE, Finsen A, et al. (2012). Inflammatory cytokines in heart failure: mediators and markers. Cardiology 122: 23-35.

Gupta M, Martineau P, Tran T, Després JP, et al. (2012). Low-density lipoprotein cholesterol and high-sensitivity C-reactive protein lowering with atorvastatin in patients of South Asian compared with European origin: insights from the Achieve Cholesterol Targets Fast with Atorvastatin Stratified Titration (ACTFAST) study. J. Clin. Pharmacol. 52: 850-858.

Kesavan M, Sarath TS, Kannan K, Suresh S, et al. (2014). Atorvastatin restores arsenic-induced vascular dysfunction in rats: Modulation of nitric oxide signaling and inflammatory mediators. Toxicol. Appl. Pharmacol. 280: 107-116.

Klop B, Verseyden C, Ribalta J, Salazar J, et al. (2014). MTP gene polymorphisms and postprandial lipemia in familial combined hyperlipidemia: effects of treatment with atorvastatin. Clin. Investig. Arterioscler. 26: 49-57.

Mordi I, Jhund PS, Gardner RS, Payne J, et al. (2014). LGE and NT-proBNP identify low risk of death or arrhythmic events in patients with primary prevention ICDs. JACC Cardiovasc. Imaging 7: 561-569.

Nuzzolo ER, Capodimonti S, Martini M, lachininoto MG, et al. (2014). Adult and cord blood endothelial progenitor cells have different gene expression profiles and immunogenic potential. Blood Transfus. 12: s367-374.

Poolsup N, Suksomboon N, Wongyaowarat K, Rungkanchananon B, et al. (2012). Meta-analysis of the comparative efficacy and safety of pitavastatin and atorvastatin in patients with dyslipidaemia. J. Clin. Pharm. Ther. 37: 166-172.

Sarath TS, Waghe P, Gupta P, Choudhury S, et al. (2014). Atorvastatin ameliorates arsenic-induced hypertension and enhancement of vascular redox signaling in rats. Toxicol. Appl. Pharmacol. 33: 79-88.

Shafi T, Zager PG, Sozio SM, Grams ME, et al. (2014). Troponin I and NT-proBNP and the association of systolic blood pressure with outcomes in incident hemodialysis patients: the choices for healthy outcomes in caring for ESRD (CHOICE) study. Am. J. Kidney Dis. 64: 443-451.

Sukpat S, Isarasena N, Wongphoom J and Patumraj S (2013). Vasculoprotective effects of combined endothelial progenitor cells and mesenchymal stem cells in diabetic wound care: their potential role in decreasing wound-oxidative stress. Biomed. Res. Int. 2013: 459196.

Van Tassell BW, Arena RA, Toldo S, Mezzaroma E, et al. (2012). Enhanced interleukin-1 activity contributes to exercise intolerance in patients with systolic heart failure. PLoS One 7: 334-338.

$\mathrm{Xu}$ Y, Yang Y and Luo YQ (2014). Effect of atorvastatin on serum oxidative stress and $\mathrm{N}$-terminal brain natriuretic peptide 
expression in rats. Asian Pac. J. Trop. Med. 7: 398-401.

Yang Z, Lee MJ, Zhao Y and Yang CS (2012). Metabolism of tocotrienols in animals and synergistic inhibitory actions of tocotrienols with atorvastatin in cancer cells. Genes Nutr. 7: 11-18.

Zhang Q, Wang H, Yang YJ, Dong QT, et al. (2014). Atorvastatin treatment improves the effects of mesenchymal stem cell transplantation on acute myocardial infarction: The role of the RhoA/ROCK/ERK pathway. Int. J. Cardiol. 176: 670-679. 